Original Research

\title{
An Alternative Model of Care for the Treatment of Adolescent Athletes with Extension-Based Low Back Pain: A Pilot Study.
}

\author{
Mitchell Selhorst, DPT, PhD, OCS ${ }^{1}$, Richard Rodenberg, MD², Nick Padgett, DPT, OCS ${ }^{1}$, Anastasia Fischer, MD, FACSM \\ ${ }^{2}$, Reno Ravindran, MD², James MacDonald, MD, MPH ${ }^{2}$ \\ 1 Sports and Orthopedic Physical Therapy, Nationwide Children's Hospital, ${ }^{2}$ Division of Sports Medicine, Nationwide Children's Hospital \\ Keywords: spondylolysis, low back pain, athlete, adolescent \\ https://doi.org/10.26603/001c.18715
}

\section{International Journal of Sports Physical Therapy}

Vol. 16, Issue 1, 2021

\section{Background and Purpose}

Half of adolescent athletes report low back pain (LBP) and there is a significant risk of vertebral injury in this population. The current model of care for adolescent athletes with LBP is to first confirm a medical diagnosis of spondylolysis which frequently requires advanced imaging. However, routine use of advanced imaging increases cost, delays treatment, and can expose the athlete to radiation.

\section{Purpose}

The purpose of this pilot study was to assess the viability of a physical therapist guided functional progression program to manage low back pain (LBP) in adolescent athletes.

\section{Study Design}

Non-randomized, controlled clinical trial.

\section{Methods}

Sixteen adolescents ( $15 \pm 1.8$ years, $50 \%$ female) with extension-based LBP were assigned to the biomedical model or physical therapy first model. The biomedical model sought to determine a spondylolysis diagnosis to guide treatment. In the physical therapy first model, patients began early therapeutic exercise and their ability to functionally progress determined the course of care. Dependent variables were change in Micheli Function Score, use of imagining, days out of sport, and ability to return to sport. Adverse events were monitored in order to assess safety. Descriptive statistics were completed to assess the viability of the alternative model.

\section{Results}

Both models had similar improvements in pain and function. The physical therapy first model reduced use of advanced imaging by $88 \%$ compared to the biomedical model. Patients in the biomedical model who did not sustain a vertebral injury returned to sport sooner than the physical therapy first model (3.4 days versus 51 days), while those with a vertebral injury took longer in the current model (131 days versus 71 days). All of the patients in the physical therapy first model and $88 \%$ of patients in the current model made a full return to sport. Two adverse events occurred in the biomedical model, and none were noted in the physical therapy first model.

\section{Conclusion}

This pilot study demonstrated that the physical therapist guided functional progression

\footnotetext{
Corresponding author:

Mitchell Selhorst, DPT, PhD, OCS

Address: 6499 East Broad St. Suite 140

Columbus, OH, 43213

E-mail address: Mitchell.Selhorst@Nationwidechildrens.org

Phone: 614-355-9764
} 
program may be a viable method for treating young athletes with LBP and further research is warranted.

\title{
Level of Evidence
}

\author{
$3 \mathrm{~b}$
}

\section{INTRODUCTION}

Low back pain (LBP) is a common complaint among adolescent athletes, and the incidence is increasing. ${ }^{1}$ The most common cause of specific LBP in this population is an isthmic spondylolysis, which has previously been reported to occur as frequently as $47 \%$ in some populations of young athletes. $^{2,3}$ However, outcomes of larger studies suggest that the prevalence of spondylolysis is likely closer to 14-30\% among adolescent athletes reporting LBP. ${ }^{3,4}$ Repetitive lumbar extension and rotation motions have been associated with increased risk of spondylolysis. ${ }^{2,5}$ Sports involving these repetitive motions, such as baseball and gymnastics, have rates of spondylolysis as high as $47-58 \%$ among symptomatic athletes. ${ }^{2,3}$ Given this high prevalence, spondylolysis should be given high priority as a diagnostic hypothesis in young athletes presenting with LBP. ${ }^{6}$

The current model of care for adolescent athletes with LBP is a biomedical model which seeks to identify a specific pathoanatomical cause of LBP using advanced imaging. ${ }^{6-9}$ Imaging is necessary to accurately distinguish spondylolysis from non-specific LBP, as there is no method to reliably identify a spondylolysis using physical examination and history. ${ }^{10,11}$ The most well-known clinical test to screen for spondylolysis, the single-leg hyperextension test, is neither sensitive nor specific for detecting spondylolysis. ${ }^{12,13}$ Consequently, diagnostic imaging is frequently obtained for this population. In adolescent athletes presenting with LBP, two-view radiographs are recommended as the first initial study. ${ }^{14}$ However, the sensitivity of radiographs is low and advanced imaging is typically necessary to obtain an accurate diagnosis. ${ }^{14}$

The outcomes of adolescent athletes following treatment using the biomedical model are not ideal. ${ }^{15-17}$ Diagnosis of adolescent LBP is associated with exposure to significant radiation and high imaging costs. ${ }^{18}$ Adolescent athletes with LBP may have a worse prognosis regarding function than their nonathlete counterparts. ${ }^{19}$ Moreover, 65\% of adolescent athletes with non-specific LBP had continued pain or a recurrence of symptoms within six months. ${ }^{16}$ In patients with a spondylolysis, $42 \%$ had a poor clinical outcome and one in eight had to stop or reduce sport participation at long-term follow-up. ${ }^{15,17}$ These findings become particularly important because the more days an adolescent experiences LBP, the higher the risk for chronic LBP as an adult. ${ }^{20}$ The suboptimal clinical outcomes in adolescent athletes with LBP demonstrate the need to improve the model of care.

An additional problem with the biomedical model of care for this population is the high cost and potential radiation exposure of diagnostic imaging. ${ }^{18}$ Single photon emission computed tomography (SPECT) with computed tomography (CT) scan, long considered the gold standard for diagnosing spondylolysis, has recently fallen out of favor due to heightened concerns over the long-term risk of malignancies as- sociated with exposure of children to radiation. ${ }^{14,21}$ Magnetic resonance imaging (MRI) is becoming more popular for identifying spondylolysis, as MRI has good diagnostic accuracy without exposing the patient to ionizing radiation. ${ }^{22}$ Challenges to the use of MRI remain, however, with issues of cost, insurance coverage, access, and variable quality of imaging in different centers. In contrast to the current recommendations to first obtain a specific diagnosis, Miller et al. ${ }^{18}$ suggested that extensive imaging is neither needed nor advisable in adolescents with LBP, arguing that advanced imaging should not be performed until the patient returns with continuing or unresolved pain following physical therapy patient management. ${ }^{18}$

The majority of LBP in adolescent athletes can be divided into two groups; non-specific LBP and spondylolysis (and other bone stress injuries). ${ }^{6,7}$ The primary difference between current treatment of non-specific LBP and spondylolysis is the need for relative rest from sport. ${ }^{6,23,24}$ Thus, the authors propose a physical therapist guided functional progression program which uses pain-free functional progress in rehabilitation in order to guide care. This model has been specifically designed to address the needs of adolescent athletes with LBP and to treat adolescent athletes without the use of advanced imaging. Patients who are able to progress back to sport without pain are believed to be stable and safe enough to do so, while those having persistent pain warrant further evaluation and perhaps need a period of rest to allow their injury to heal. Subsequently, the purpose of this pilot study was to assess the viability of a physical therapist guided functional progression program to manage LBP in adolescent athletes.

\section{METHODS}

This pilot study was a non-randomized, controlled trial using a sample of convenience. Adolescent athletes with low back pain that is increased during lumbar extension presenting to participating sports medicine physicians at $\mathrm{Na}-$ tionwide Children's Hospital (Columbus, Ohio) were considered for participation. The institutional review board approved this study prior to recruitment and data collection. All patients and guardians provided written informed consent prior to participation. This study was registered at ClinicalTrials.gov (Identifier number NCT02861456).

\section{PARTICIPANTS}

Patients were eligible if they were an adolescent athlete (12-19 years) who reported acute LBP ( $<3$ months) which increased during lumbar extension. To be considered an athlete, patients had to participate in sport activity at least two times a week prior to the onset LBP. Patients were excluded if they (1) already had advanced imaging performed, (2) demonstrated red flags (bowel or bladder problems, saddle anesthesia, progressive neurological deficits, recent 


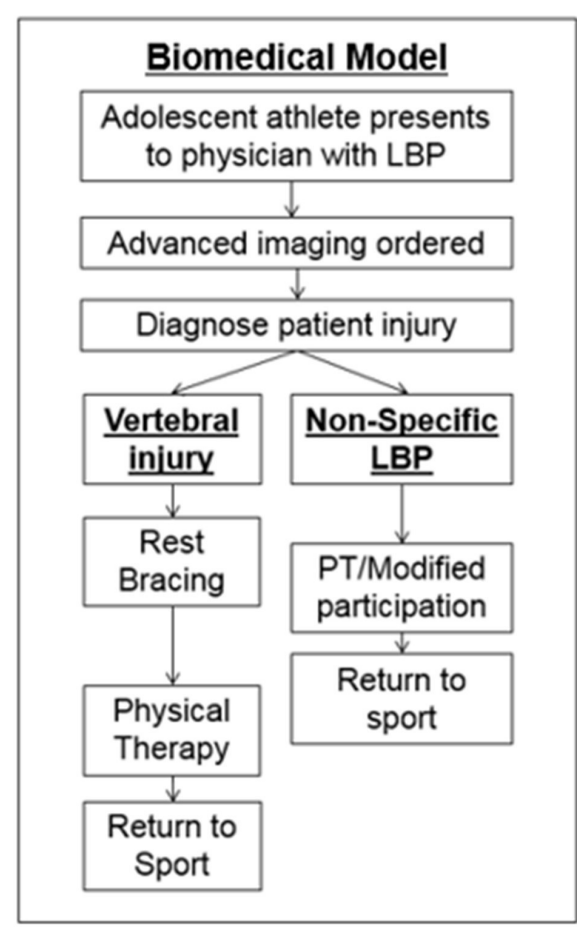

Figure 1. Models of Care Diagram

fever of infection, unexplained weight loss, unable to change symptoms with clinical testing) (3) previously rested from sport for $>4$ weeks due to LBP, (4) reported other injury or conditions which would alter the plan of care for LBP (i.e. pregnancy, concomitant ACL tear), or (5) history of lumbar surgery.

\section{TREATMENT ALLOCATION}

This study was not randomized and the intervention the patient received was based upon their co-investigating physician. Patients either received treatment based on the proposed physical therapist guided functional progression program (PT First) or a biomedical model of care (Figure 1). Consecutive patients treated by two co-investigating physicians (AF and RR) were treated using the PT First program, while patients treated by the other two co-investigating physicians (JM and RR) were treated using a biomedical model of care. Both interventions used a pragmatic approach.

\section{INTERVENTIONS}

\section{PHYSICAL THERAPIST GUIDED FUNCTIONAL PROGRESSION PROGRAM (PT FIRST)}

Patients in the PT First group had radiographs but did not immediately have advanced imaging obtained to determine diagnosis and instead were placed on rest from sport and began the physical therapist guided functional progression program. Therapy in the PT First group was performed twice a week and followed a three-phase program (Table 1). Progression through the physical therapy program was based on achieving specific criteria, as opposed to a timebased protocol. Patients performed Phase I of the physical

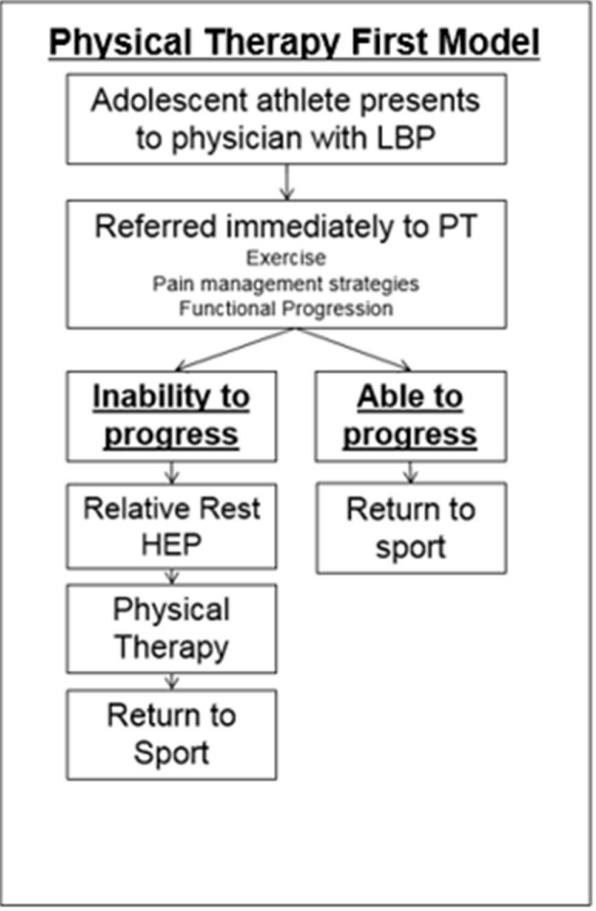

therapy program and progressed to phase II after meeting the specified criteria without an increase in pain. The criteria to progress to the next phase was assessed at each session. Patients who met the criteria to pass Phase I and II within a designated three-week period progressed into the third phase of physical therapy to begin return to sport activity. Patients who progressed into Phase III at this time were given additional physical therapy visits. Patients who progressed well in this third phase, and met the return to sport criteria, were released to return to sport and were discharged from physical therapy with a home exercise program. Patients who failed to meet the criteria of Phase I and II within three weeks and Phase III after a total of five weeks were considered to have an inability to progress.

Patients who had an inability to progress during the initial course of physical therapy were either treated as a presumed spondylolysis or had advanced imaging performed as a shared decision of the patient, family, and physician. Patients who had advanced imaging ordered after failing to meet the criteria were treated based upon the imaging results. Patients treated as a presumed vertebral injury were placed on rest for two months from all activities except activities of daily living and their physical therapy home exercise program. Following the two months of rest the patient then completed physical therapy care before returning to sport.

\section{BIOMEDICAL MODEL OF CARE}

Adolescents with LBP in the control group received care based upon the biomedical model. The biomedical model consisted of the physician first attempting to determine if the adolescent athlete had a spondylolysis or non-specific LBP. Advanced imaging was obtained to diagnose the injury 
Phase I Protected Phase

\begin{tabular}{|l|l|}
\hline $\begin{array}{l}\text { Core strengthening in neutral spine } \\
\text { Treat directional preference if identified } \\
\text { Hip strengthening }\end{array}$ & \multicolumn{1}{c|}{$\begin{array}{c}\text { Criteria to begin Phase II } \\
\text { Peri-scapular strengthening }\end{array}$} \\
$\begin{array}{l}\text { Flexibility exercises } \\
\text { Manual Therapy as needed }\end{array}$ & $\begin{array}{l}\text { 1. Good core stability in neutral spine during } \\
\text { exercises (clinician judgement) }\end{array}$ \\
Modalities for pain (use sparingly) & $\begin{array}{l}\text { 2. Pain free repeated standing extension } \times 10 \\
\text { 3. Pain free repeated standing flexion } \times 10\end{array}$ \\
\cline { 2 - 2 }
\end{tabular}

Phase II Functional Exercise Phase
\begin{tabular}{|l|l|}
\hline $\begin{array}{l}\text { Core strengthening in functional range } \\
\text { Hip and peri-scapular strengthening }\end{array}$ & \multicolumn{1}{c|}{ Criteria to begin Phase III } \\
$\begin{array}{l}\text { Flexibility exercises } \\
\text { Manual Therapy (use sparingly) }\end{array}$ & $\begin{array}{l}\text { 1. } 0 \% \text { score on ADL and Pain subsections (B } \\
\text { and C) of Micheli Functional Scale }\end{array}$ \\
$\begin{array}{l}\text { Light Running } \\
\text { Jumping }\end{array}$ & $\begin{array}{l}\text { 2. Pain free for } 2 \text { consecutive visits during } \\
\text { functional extension, rotation, and flexion } \\
\text { exercises }\end{array}$ \\
\hline
\end{tabular}

Phase III Return to Sport Phase

\begin{tabular}{|l|l|}
\hline $\begin{array}{l}\text { Return to sport activity with focus on } \\
\text { functional return to all aspect of sport. }\end{array}$ & \multicolumn{1}{c|}{$\begin{array}{c}\text { Return to sport criteria } \\
\text { 1. Pain free at end range of all lumbar motions }\end{array}$} \\
& $\begin{array}{l}\text { 2. Completed 2 weeks of return to sport } \\
\text { activity in physical therapy without pain. } \\
\text { 3. } 0 \% \text { score on Micheli Functional Scale }\end{array}$ \\
\hline
\end{tabular}

\section{TABLE 1. Physical Therapy First- Physical Therapy Program ${ }^{1}$}

1. d'Hemecourt PA, Zurakowski D, d'Hemecourt CA, et al. Validation of a new instrument for evaluating low back pain in the young athlete. Clin J Sport Med. 2012;22(3):244-8.

when the physician had sufficient suspicion of a vertebral injury in the athlete with low back pain. If there was no identifiable cause of the patient's LBP, the patient was sent to physical therapy and was allowed to progress to sport immediately. If the patient was diagnosed with a bony or spondylolytic injury, care would consist of a preliminary two to three month rest from activity, bracing if the physician thought it was necessary, followed by four to six weeks of physical therapy. Physical therapy was performed twice per week and interventions were individualized based on the patient's presentation.

\section{OUTCOMES}

Use of advanced imaging, cost of care, and clinical outcomes were the main outcomes of interest. Clinical outcomes included pain, function, ability to return to sport, and time to return to sport. Pain and function were assessed at baseline and discharge using the Micheli Functional Scale (MFS), as traditional adult patient reported outcome measures are notably less reliable in high functioning individuals. ${ }^{25-27}$ The MFS is a self-report measure designed to assess pain and functional ability of adolescent athletes with LBP on a 0-100 scale with 0 being no disability and 100 representing maximum disability. This scale has been found to have validity (concurrent validity $r=0.90$ ), and reliability (item reliability $\alpha=0.79-0.90$ ) in young athletes with LBP. ${ }^{28,29}$ The patient's ability to participate in sport was assessed using a self-report question reporting one of the following: 1) I am at the same or higher level of sport than I was prior to treatment, 2) I am still in my sport but at a lower level due to my low back injury, 3) I am currently un- able to participate in sport due to my low back injury or 4) I am currently not participating in sport, but my low back was not a factor. Time to return to sport was the number of days from when the physician instructed the patient to rest from sport to when the patient was cleared to return to sport. If the patient was not instructed to rest from sport, time to return to sport was zero days.

\section{SAFETY}

Safety of the PT First program was assessed by monitoring adverse events in both groups. An adverse event was defined as 1) lumbar symptoms increasing enough to cause an unplanned visit to a physician, 2) the patient being placed on hold from therapy during the episode of care due to increased low back pain, or 3) there was a significant delay (>4 weeks) in diagnosis of non-mechanical low back pain which could have been made by use of advanced imaging.

\section{BLINDING}

No blinding was performed in this pilot trial as it was not feasible to blind either the clinicians or the patient to the treatment cohort.

\section{SAMPLE SIZE}

A sample of eight participants in each group was recruited for this pilot trial. This number was deemed sufficient to provide estimates of outcomes to guide future research studies. 
TABLE 2. Demographics and Baseline Variables. Data are presented as means (SD) or numbers (\%)

\begin{tabular}{|l|l|l|l|}
\hline & \multicolumn{1}{|c|}{$\begin{array}{c}\text { All Patients } \\
(\mathrm{n}=16)\end{array}$} & \multicolumn{1}{|c|}{$\begin{array}{c}\text { Physical Therapy First Model } \\
(\mathrm{n}=8)\end{array}$} & $\begin{array}{c}\text { Biomedical Model } \\
(\mathrm{n}=8)\end{array}$ \\
\hline Age & $15.0 \pm 1.8$ & $14.5 \pm 12.1$ & $15.5 \pm 1.4$ \\
\hline Gender (\% female) & $8(50 \%)$ & $5(62 \%)$ & $3(38 \%)$ \\
\hline Micheli Functional scale & $46.8 \pm 14.5$ & $40.0 \pm 11.3$ & $53.5 \pm 14.8$ \\
\hline Unable to play & $13(81 \%)$ & $6(75 \%)$ & $7(88 \%)$ \\
\hline Pain & $5.2 \pm 2.3$ & $4.8 \pm 2.5$ & $5.6 \pm 2.2$ \\
\hline
\end{tabular}

TABLE 3. Course of Care. Data are presented as means (SD)

\begin{tabular}{|c|c|c|c|}
\hline & $\begin{array}{l}\text { All Patients } \\
\quad(n=16)\end{array}$ & $\begin{array}{l}\text { Physical Therapy First Model } \\
\qquad(\mathrm{n}=8)\end{array}$ & $\begin{array}{l}\text { Biomedical Model } \\
(n=8)\end{array}$ \\
\hline Duration of Care (days) & $62 \pm 32$ & $59 \pm 21$ & $65 \pm 41$ \\
\hline Physical therapy visits & $8.9 \pm 4.6$ & $11.5 \pm 3.2$ & $6.5 \pm 4.6$ \\
\hline Physician visits & $3.2 \pm 1.8$ & $2.8 \pm 1.2$ & $3.6 \pm 2.3$ \\
\hline
\end{tabular}

\section{DATA ANALYSIS}

To assess the viability of a physical therapist guided functional progression program to treat young athletes with extension-oriented LBP descriptive statistics of the patient demographics and outcome variables were calculated using SPSS 24. This was a pilot study and is underpowered for inferential statistics, therefore only descriptive statistics were used to assess the viability of the PT first program. ${ }^{30}$

\section{RESULTS}

Sixteen patients were recruited for this pilot study and were assigned to either the biomedical model $(n=8)$ or the PT First program $(n=8)$ group. Eligible patients were recruited from August through December of 2016. Twenty-eight consecutive patients who presented to the participating sports medicine physicians with low back pain were screened for inclusion (Figure 2). Baseline variables were similar between the two models of care based upon comparison of descriptive statistics (Table 2). Eight advanced imagining procedures were performed in the biomedical model group and only one was performed in the PT First group. In the biomedical model group, five of the patients (63\%) were diagnosed with non-specific low back pain, two of the patients (25\%) were diagnosed with spondylolysis and one patient (12\%) with a herniated disc. A definitive diagnosis in the PT First program was not made for patients (as per protocol), but based on the patient's ability to improve, six (75\%) were able to make a full return to sport and activity directly from the program. Two patients (25\%) did not progress through the program without pain. Advanced imaging was obtained with one patient, while the other was treated as a presumed vertebral injury and requiring a period of rest to make a full return to sport.

The course of care differed between both models of care (Table 3). Use of advanced imaging was $88 \%$ lower in the PT
First program. On average, nearly twice as many physical therapy visits were used in the PT First program, while slightly more physician visits were used in the biomedical model. Both models of care had a similar duration of care. The median billed cost for the PT First program was 19\% lower than in the biomedical model (\$3885.00 versus \$4774.00).

Both models of care made similar improvements in clinical outcomes (Table 4 ). Overall, $94 \%$ of the patients between the groups $(n=15)$ were able to make a full return to sport in an average of 49 days ( \pm 43 days). In this pilot study, the duration of care for vertebral injuries (101 \pm 20 days) was longer than for non-specific LBP (49 \pm 23 days). Patients in the biomedical model group with non-specific LBP were cleared to return to sport much sooner than the PT First program (3.4 days versus 51 days). However, those with a diagnosed vertebral injury took longer to be cleared to return to sport in the biomedical model group than those with a presumed vertebral injury in the PT First group (131 days versus 71 days).

Two adverse events were noted in the biomedical model group, and none occurred in the PT First group. The relative risk of adverse events was not calculated due to the nature of this pilot study. One patient initially had a SPECT scan which revealed no vertebral injury, and physical therapy was prescribed. The patient did not attend her physical therapy evaluation, and two weeks later had a significant worsening of LBP with tingling in the right leg. Clinical examination revealed no myotomal weakness of the lower extremities, intact deep tendon reflexes and decreased sensation to light touch in the right medial foot. The patient's co-investigating physician ordered an MRI which revealed a herniated disc at L4. The patient and family sought a referral to neurology and opted to have a microdiscectomy. She was the only patient who reported being unable to return to sport. Another patient was diagnosed initially with a bilateral spondylolysis at L4 and started physical therapy after 10 weeks of rest, had a significant worsening of LBP during 
TABLE 4. Clinical Outcomes. Data are presented as means (SD)

\begin{tabular}{|l|l|l|l|}
\hline & \multicolumn{1}{|c|}{$\begin{array}{c}\text { All Patients } \\
(\mathrm{n}=16)\end{array}$} & \multicolumn{1}{|c|}{$\begin{array}{c}\text { Physical Therapy First Model } \\
(\mathrm{n}=8)\end{array}$} & $\begin{array}{c}\text { Biomedical Model } \\
(\mathrm{n}=8)\end{array}$ \\
\hline Change in Micheli & $33.5 \pm 17.6$ & $30.5 \pm 14.9$ & $36.5 \pm 20.5$ \\
\hline Change in Pain & $4.3 \pm 2.4$ & $4.1 \pm 2.3$ & $4.4 \pm 2.6$ \\
\hline Full return to sport & $15(94 \%)$ & $8(100 \%)$ & $7(88 \%)$ \\
\hline Global Rating of Change & $5.4 \pm 1.3$ & $5.6 \pm 1.2$ & $5.1 \pm 1.4$ \\
\hline
\end{tabular}

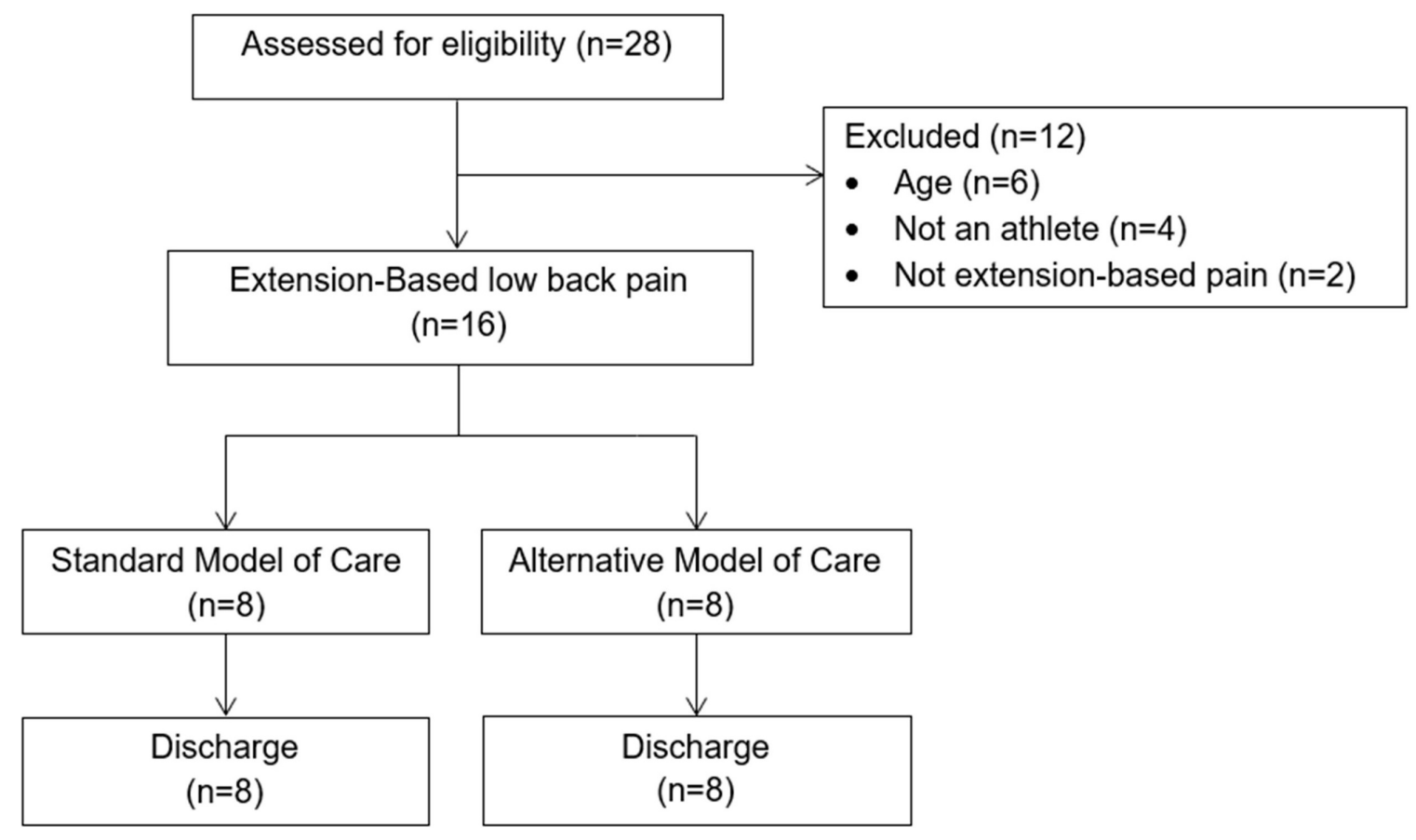

Figure 2. Participant Flow Diagram

care requiring her to stop participation. She subsequently had repeat advanced imaging which revealed no additional significant findings.

\section{DISCUSSION}

The purpose of this pilot study was to assess an alternative way to manage LBP in adolescent athletes in a safe manner without exposing the patient to unnecessary and expensive testing. This study demonstrated that by using the physical therapist guided functional progression (PT First) program there is the potential to safely reduce the need for advanced imaging by $88 \%$ in adolescents with LBP. Although a specific diagnosis was not obtained in the PT First group, all of the participants were able to return to sport, despite two patients (25\%) requiring a period of rest from the treatment protocol before returning to progress back to sport. Only one of these two patients required advanced imaging. Early advanced imaging, as seen in the biomedical model, resulted in a faster return to sport ( 3.4 days versus 51 days) in individuals who did not sustain a vertebral injury. This quick return is likely due to physician comfort in the knowl- edge that the spine was anatomically 'normal' allowing the physician to release the patient earlier to full play while doing therapy at the same time to work on mechanics and functional issues. However, this knowledge comes at a high monetary cost.

Adolescent LBP can be intimidating for clinicians to care for in clinical practice and dogma for pediatricians and trainees has been that most LBP in this population has an identifiable diagnosis. ${ }^{31,32}$ This thinking has been perpetuated because up to $47 \%$ of adolescent athletes were reported to have spondylolysis when presenting with extension-based LBP. ${ }^{2}$ Moreover, medical trainees are taught that adolescents with LBP lasting longer than two to three weeks should be investigated; prompting radiographs and often times advanced imaging. Both single photon emission computed tomography (SPECT) with computed tomography (CT) and magnetic resonance imaging (MRI) can be utilized to identify spondylolysis, however, advanced imaging comes at a significant cost and is not without risks. There is no one test that fits all situations and is available to answer all questions about the potential etiology of pain in the adolescent spine. MRI is an appealing test due to the improving diagnostic accuracy without ionizing radiation, but 
many consider SPECT with CT the gold standard in identifying stress reaction or fracture of the posterior elements of the lumbar spine. ${ }^{33}$ Campbell et ${ }^{34}$ al looked at MRI versus SPECT with CT in a diagnostic validation study and found MRI is an accurate means of demonstrating a normal pars, acute complete defects, and chronic established defects but was limited in its ability to accurately diagnose stress reaction and incomplete defects; prompting some clinicians to still utilize SPECT in the care of their patients. Further, both MRI and SPECT with CT come at a significant monetary cost.

Recent research has challenged the thought process that there is frequently an identifiable cause for LBP in adolescent athletes. ${ }^{18,35,36}$ A high quality prospective study of 73 pediatric LBP patients followed for two years found that nearly $80 \%$ had no definitive diagnosis. A retrospective study by Miller et $\mathrm{al}^{18}$ found that a majority of school age children presenting to a tertiary referral center to see a pediatric orthopedic specialist had undiagnosed mechanical LBP. Of 2846 patients followed over an 8-year period, $75.9 \%$ were determined to have undiagnosed mechanical LBP. The majority of patients diagnosed with non-specific mechanical LBP had $\leq 2$ follow-up visits, indicating that recovery was obtained quickly in these patients. ${ }^{18}$ Similar to reports by Miller, ${ }^{18}$ Houghton, ${ }^{36}$ and Shah, ${ }^{35}$ imaging in the current pilot study found an identifiable etiology in three of eight participants (2 spondylolysis and 1 herniated disc). Pursuit of a specific etiology comes at significant monetary cost given that $100 \%$ PT First program participants were able to ultimately advance back to full sports participation without advanced imaging.

Based on the results of this pilot study, the authors suggest that LBP in adolescent athletes can be successfully addressed by basing treatment and progression on functional outcomes without utilizing expensive imaging modalities that often times do not reveal a true etiology for the patients' LBP.

\section{LIMITATIONS}

This pilot study is not without limitations, primarily the lack of blinding and randomization to the models of care. Blinding the treating clinicians and patients to the assigned model of care was impractical due to the nature of the planned treatments. The non-randomized design potentially biased the results of this pilot study, particularly with the slightly higher levels of pain and dysfunction at baseline noted in the biomedical model group. The authors plan a future larger randomized study to reduce study bias and assess clinical effectiveness of the PT First program.

\section{CONCLUSION}

The proposed physical therapist guided functional progression (PT First) program may be a viable method for treating adolescent athletes with low back pain, particularly when there are financial concerns, concerns for radiation exposure, or the athlete is in their off-season. However, the authors admit that the PT First program produced a much longer duration of therapy and was associated with a longer return to play timeline compared to the biomedical model where imaging was done. A speedy return-to-play should be considered in an in-season or high-level adolescent athlete if it can be done without patient harm. The authors of this study plan to continue to study the physical therapist guided functional progression (PT First) program protocol through a larger randomized controlled trial.

\section{CONFLICTS OF INTEREST}

The authors have no conflicts of interest to report

Submitted: November 18, 2019 CDT, Accepted: June 16, 2020 CDT 


\section{REFERENCES}

1. National Council of Youth Sports. Report on Trends and Participation in Organized Youth Sports.; 2008. htt p://www.ncys.org/publications/2008-sports-participa tionstudy.php. Accessed March 16, 2018.

2. Micheli LJ, Wood R. Back pain in young athletes. Significant differences from adults in causes and patterns. Arch Pediatr Adolesc Med. $1995 ; 149(1): 15-18$.

3. Selhorst M, Fischer A, MacDonald J. Prevalence of spondylolysis in symptomatic adolescent athletes: An assessment of sport risk in nonelite athletes. Clin J Sport Med. 2019;29(5):421-425. doi:10.1097/jsm.0000 $\underline{000000000546}$

4. Rossi F, Dragoni S. The prevalence of spondylolysis and spondylolisthesis in symptomatic elite athletes: radiographic findings. Radiography. 2001;7(1):37-42. doi:10.1053/radi.2000.0299

5. Cavalier R, Herman MJ, Cheung EV, Pizzutillo PD. Spondylolysis and spondylolisthesis in children and adolescents: I. Diagnosis, natural history, and nonsurgical management. J Am Acad Orthop Surg. 2006;14(7):417-424.

6. De Luigi AJ. Low back pain in the adolescent athlete. Phys Med Rehabil Clin N Am. 2014;25(4):763-788. doi:10.1016/i.pmr.2014.06.004

7. Standaert CJ. Low back pain in the adolescent athlete. Phys Med Rehabil Clin N Am. 2008;19(2):287-304, ix. doi:10.1016/i.pmr.2008.01.00 $\underline{2}$

8. Standaert CJ, Herring SA. Expert opinion and controversies in sports and musculoskeletal medicine: the diagnosis and treatment of spondylolysis in adolescent athletes. Arch Phys Med Rehabil. 2007;88(4):537-540. doi:10.1016/i.apmr.200 7.01.007

9. Syrmou E, Tsitsopoulos PP, Marinopoulos D, Tsonidis C, Anagnostopoulos I, Tsitsopoulos PD. Spondylolysis: a review and reappraisal. Hippokratia. 2010;14(1):17-21.

10. Alqarni AM, Schneiders AG, Cook CE, Hendrick PA. Clinical tests to diagnose lumbar spondylolysis and spondylolisthesis: A systematic review. Phys Ther Sport. 2015;16(3):268-275. doi:10.1016/j.ptsp.2014.1 $\underline{2.005}$
11. Grodahl LH, Fawcett L, Nazareth M, et al. Diagnostic utility of patient history and physical examination data to detect spondylolysis and spondylolisthesis in athletes with low back pain: A systematic review. Man Ther. 2016;24:7-17. doi:10.10 16/i.math.2016.03.011

12. Masci L, Pike J, Malara F, Phillips B, Bennell K, Brukner P. Use of the one-legged hyperextension test and magnetic resonance imaging in the diagnosis of active spondylolysis. $\mathrm{Br} J$ Sports $\mathrm{Med}$. 2006;40(11):940-946; discussion 946. doi:10.1136/bjs m.2006.030023

13. Gregg CD, Dean S, Schneiders AG. Variables associated with active spondylolysis. Phys Ther Sport. 2009;10(4):121-124. doi:10.1016/j.ptsp.2009.08.001

14. Tofte JN, CarlLee TL, Holte AJ, Sitton SE, Weinstein SL. Imaging pediatric spondylolysis: A systematic review. Spine. 2017;42(10):777-782. doi:1 $0.1097 /$ brs. 0000000000001912

15. Sousa T, Skaggs DL, Chan P, et al. Benign natural history of spondylolysis in adolescence with midterm follow-up. Spine Deform. 2017;5(2):134-138. doi:10.10 16/j.jspd.2016.10.005

16. Selhorst M, Selhorst B. Lumbar manipulation and exercise for the treatment of acute low back pain in adolescents: a randomized controlled trial. J Man Manip Ther. 2015;23(4):226-233. doi:10.1179/204261 $\underline{8614 \mathrm{y} .0000000099}$

17. Selhorst M, Fischer A, Graft K, et al. Long-term clinical outcomes and factors that predict poor prognosis in athletes after a diagnosis of acute spondylolysis: A retrospective review with telephone follow-up. J Orthop Sports Phys Ther.

2016;46(12):1029-1036. doi:10.2519/jospt.2016.7028

18. Miller R, Beck NA, Sampson NR, Zhu X, Flynn JM, Drummond D. Imaging modalities for low back pain in children: a review of spondyloysis and undiagnosed mechanical back pain. J Pediatr Orthop. 2013;33(3):282-288. doi:10.1097/BPO.0b013e318287f $\underline{\mathrm{ffb}}$

19. Fritz JM, Clifford SN. Low back pain in adolescents: a comparison of clinical outcomes in sports participants and nonparticipants. J Athl Train. 2010;45(1):61-66. doi:10.4085/1062-6050-45.1.61 
20. Hestbaek L, Leboeuf-Yde C, Kyvik KO, Manniche C. The course of low back pain from adolescence to adulthood: eight-year follow-up of 9600 twins. Spine. 2006;31(4):468-472. doi:10.1097/01.brs.0000199958.0 4073.d9

21. Miglioretti DL, Johnson E, Williams A, et al. The use of computed tomography in pediatrics and the associated radiation exposure and estimated cancer risk. JAMA Pediatr. 2013;167(8):700-707. doi:10.1001/ jamapediatrics.2013.311

22. Kobayashi A, Kobayashi T, Kato K, Higuchi H, Takagishi K. Diagnosis of radiographically occult lumbar spondylolysis in young athletes by magnetic resonance imaging. Am J Sports Med. 2013;41(1):169-176. doi:10.1177/0363546512464946

23. El Rassi G, Takemitsu M, Glutting J, Shah SA. Effect of sports modification on clinical outcome in children and adolescent athletes with symptomatic lumbar spondylolysis. Am J Phys Med Rehabil. 2013;92(12):1070-1074. doi:10.1097/PHM.0b013e318 296da7e

24. Standaert CJ. Spondylolysis in the adolescent athlete. Clin J Sport Med. 2002;12(2):119-122.

25. Brodke DS, Goz V, Lawrence BD, Spiker WR, Neese A, Hung M. Oswestry Disability Index: a psychometric analysis with 1,610 patients. Spine $J$.

2017;17(3):321-327. doi:10.1016/j.spinee.2016.09.020

26. Hall AM, Maher CG, Latimer J, Ferreira ML, Costa LO. The patient-specific functional scale is more responsive than the Roland Morris disability questionnaire when activity limitation is low. Eur Spine J. 2011;20(1):79-86. doi:10.1007/s00586-010-15 21-8

27. Kent P, Grotle M, Dunn KM, Albert HB, Lauridsen $\mathrm{HH}$. Rasch analysis of the 23-item version of the Roland Morris Disability Questionnaire. J Rehabil Med. 2015;47(4):356-364. doi:10.2340/16501977-1935
28. d'Hemecourt PA, Zurakowski D, d'Hemecourt CA, et al. Validation of a new instrument for evaluating low back pain in the young athlete. Clin J Sport Med. 2012;22(3):244-248. doi:10.1097/JSM.0b013e318249a 3ce

29. MacDonald JP, d'Hemecourt PA, Micheli LJ. The reliability and validity of a pediatric back outcome measure. Clin J Sport Med. 2016;26(6):490-496. doi:1 $\underline{0.1097 / \text { jsm.0000000000000282 }}$

30. Kannan S, Gowri S. Pilot studies: Are they appropriately reported? Perspect Clin Res. 2015;6(4):207-210. doi:10.4103/2229-3485.167097

31. Ginsburg GM, Bassett GS. Back pain in children and adolescents: Evaluation and differential diagnosis. J Am Acad Orthop Surg. 1997;5(2):67-78.

32. Waicus KM, Smith BW. Back injuries in the pediatric athlete. Curr Sports Med Rep. 2002;1(1):52-58.

33. Dhouib A, Tabard-Fougere A, Hanquinet S, Dayer R. Diagnostic accuracy of MR imaging for direct visualization of lumbar pars defect in children and young adults: a systematic review and meta-analysis. Eur Spine J. 2018;27(5):1058-1066. doi:10.1007/s0058 6-017-5305-2

34. Campbell RS, Grainger AJ, Hide IG, Papastefanou S, Greenough CG. Juvenile spondylolysis: a comparative analysis of CT, SPECT and MRI. Skeletal Radiol. 2005;34(2):63-73. doi:10.1007/s00256-004-08 $\underline{78-3}$

35. Shah SA, Saller J. Evaluation and Diagnosis of Back Pain in Children and Adolescents. J Am Acad Orthop Surg. 2016;24(1):37-45. doi:10.5435/JAAOSD-14-00130

36. Houghton KM. Review for the generalist: evaluation of low back pain in children and adolescents. Pediatr Rheumatol Online J. 2010;8:28. do $\mathrm{i}: 10.1186 / 1546-0096-8-28$ 